\title{
Towards label-free mid-infrared protein assays: in-situ formation of bare gold nanoparticles for surface enhanced infrared absorption spectroscopy of bovine serum albumin
}

\author{
Ángela Inmaculada López-Lorente ${ }^{1} \cdot$ Pei Wang ${ }^{1} \cdot$ Boris Mizaikoff $^{1}$
}

Received: 6 July 2016 / Accepted: 24 November 2016

(C) Springer-Verlag Wien 2016

\begin{abstract}
Surface enhanced infrared absorption spectroscopy (SEIRAS) with attenuated total reflection (ATR) configuration has been used to determine bovine serum albumin (BSA) protein adsorbed onto bare gold nanoparticles (AuNPs). The AuNPs forming the SEIRA-active substrate were directly synthesized inside the ATR liquid cell taking advantage of stainless steel assisted synthesis via the walls composing the ATR compartment. The formation of the AuNPs can be directly monitored via the enhancement of the corresponding water absorption features. The absorbance of BSA at the AuNPs deposited onto the Si ATR waveguide is significantly enhanced when compared to bare $\mathrm{Si}$, thereby improving the sensitivity of detection. Apparently, the presence of the AuNPs layer at the ATR waveguide surface at the measured conditions does not affect the secondary structure of the protein. Measurements were performed in water and confirmed in deuterium oxide, which significantly reduces the strongly absorbing mid-infrared background of water in the spectral regime of interest. The limits of detection achieved for BSA protein analysis in water and deuterium oxide media are 1.93 and $4.15 \mathrm{mg} \cdot \mathrm{L}^{-1}$, respectively, and the precisions at a $250 \mathrm{mg} \cdot \mathrm{L}^{-1}$ concentration are $11.9 \%$ and $8.1 \%$, respectively.
\end{abstract}

Electronic supplementary material The online version of this article (doi:10.1007/s00604-016-2031-0) contains supplementary material, which is available to authorized users.

Ángela Inmaculada López-Lorente angela.lopez@uni-ulm.de

1 Institute of Analytical and Bioanalytical Chemistry, Ulm University, 89081 Ulm, Germany
Keywords ATR · SEIRA · FTIR · Scanning electron microscopy $\cdot$ MIR waveguide $\cdot$ Evanescent wave $\cdot$ BSA Adsorption isotherm

\section{Introduction}

Mid-infrared (MIR) spectroscopy investigates the interaction of MIR photons with molecules via the excitation of vibrational and rotational modes with inherent molecular selectivity. Hence, an ideal platform for optical sensing applications including the analysis of proteins is provided [1]. The MIR characteristics of proteins include bands that arise predominantly from vibrations within the peptide backbone such as the amide I and amide II vibrations among others. The amide I band $\left(1600-1700 \mathrm{~cm}^{-1}\right)$ is related to $\mathrm{C}=\mathrm{O}$ stretching vibration, whereas the amide II band $\left(1500-1600 \mathrm{~cm}^{-1}\right)$ is associated with the $\mathrm{CN}$ stretching and the $\mathrm{NH}$ in-plane bending [2].

Infrared attenuated total reflection (IR-ATR) spectroscopy is a commonly used analytical technique to study proteins in general, and more specifically, protein conformation and aggregation. An evanescent field generated by electromagnetic radiation totally internally reflected along the waveguide/ sample interface penetrates with exponentially decaying field strength into the adjacent medium. Thereby, MIR photons may interact with molecular species present within few micrometers of the ATR waveguide surface, i.e., the penetration depth of the evanescent field. Complementarily, surface enhanced infrared absorption spectroscopy (SEIRAS) has become a powerful tool to study adsorption and desorption processes, for example of biological molecules such as proteins at noble metal films. Hartstein et al. [3] reported for the first time on the so-called surface enhanced infrared absorption effect occurring at such noble metal surfaces. The observed enhancement is attributed to both an electromagnetic 
enhancement, which increases the local electric field at the surface, and a chemical enhancement mechanism resulting from chemical interactions between molecules and metallic nanoparticles [4].

SEIRAS measurements are usually carried out at individual metal islands or metal island films. The achieved signal enhancement depends on the size, shape, and particle density of metal island film [5]. Thus, templates such as periodic particle-array films [6] prepared by nanosphere lithography have been used for obtaining a controlled SEIRA-active surface morphology. Alternatively, wet-chemical deposition of nano-islands noble metal films have been reported [7].

We propose herein the in-situ synthesis of bare gold nanoparticles as SEIRA substrate inside the ATR liquid cell taking advantage of the stainless steel walls of the compartment, as pioneered by the team of Mizaikoff [8]. In this process, stainless steel acts as a reducing agent, which leads to the inherent formation of a thin layer of AuNPs deposited at the ATR waveguide surface within the evanescent field [8]. This facile and rapid procedure for the in-situ formation of AuNPs at a substrate surface facilitates establishing a renewable SEIRAsurface prior to each measurement. In addition, the formation of the SEIRA active layer can be readily monitored in-situ by tracking the enhancement produced on the corresponding water absorption bands. During previous studies our research team has demonstrated the suitability of the stainless steel mediated bare AuNPs for surface enhanced Raman spectroscopy (SERS) $[9,10]$ improving the determination of carbon nanotubes [11]. Herein, we report investigations on the suitability of these in-situ formed bare AuNPs for enhanced protein analysis in the MIR region.

ATR-SEIRAS has been used to monitor the adsorption kinetics of bovine hemoglobin (BHb) at an AuNP film deposited at a hemispherical silicon surface via a galvanic exchange reaction [12], as well as for monitoring the oriented assembly of strep-tagged membrane proteins at a gold surface [13]. In addition, this approach has also been applied for monitoring protein-protein binding interactions [14]. Moreover, the interaction [15], adsorption [16], and dynamics [17] of cytochrome $\mathrm{c}$ have been also investigated via SEIRAS.

Bovine serum albumin (BSA) is a protein composed of 583 amino acids with a molecular weight of approximately 66.5 $\mathrm{KDa}$. The secondary structure of BSA is dominated by helical structures $(67 \%)$ along with turn and extended chain configurations [18]. BSA protein has a rather high affinity towards gold nanoparticles, since albumin in general presents an external thiol group available for conjugation to the AuNP surface [19]. Binding of BSA to citrate-coated AuNPs has been described [20], while binding of BSA to bare gold is nearly twice as pronounced [21]. Herein, the determination of BSA protein has been advanced by taking advantage of the affinity of the protein to in-situ synthesized and readily renewable bare AuNPs present within the evanescent field of a multi- reflection IR-ATR waveguide. Thereby, the potential for a label-free mid-infrared protein conformation assay technology with enhanced sensitivity is clearly evident.

\section{Materials and methods}

\section{Reagents and materials}

Hydrogen tetrachloroaurate (III) trihydrate ACS $99.99 \%$ from Alfa Aesar (Karlsruhe, Germany, www.alfa.com) was used to synthesize the gold nanoparticles. Albumin from bovine serum (BSA) (Sigma A9418) was provided by Sigma-Aldrich Co. (St. Louis, MO, www.sigmaaldrich.com). $10 \mathrm{mg} \cdot \mathrm{mL}^{-1}$ stock solution of the protein was prepared in ultrapure water. Deuterium oxide $\left(\mathrm{D}_{2} \mathrm{O}, 99.9 \%\right)$ was provided by Deutero GmbH (Kastellaun, Germany, www.deutero.de).

\section{In-situ synthesis of bare gold nanoparticles inside the ATR liquid cell}

Gold nanoparticles were directly synthesized inside the liquid cell of the ATR unit by taking advantage of the stainless steel walls of the cell, similarly to the procedure previously described by our research team $[8,9] .20 \mu \mathrm{L}$ of a $0.1 \%$ tetrachloroauric acid solution were placed into the ATR liquid cell, where the spontaneous formation of gold nanoparticles occurs. The reaction was maintained for $15 \mathrm{~min}$ prior to the protein measurements. During this period, the obtained AuNPs inherently sedimented at the ATR waveguide surface. The synthesis of the AuNPs can be directly monitored by following the signal enhancement on the water absorption features resulting from the corresponding SEIRA effect.

\section{Surface enhanced infrared absorption studies}

SEIRA spectra of protein samples were recorded using a Bruker Vertex 70 FT-IR spectrometer (Bruker Optik, GmbH, Ettlingen, Germany) equipped with an attenuated total reflection unit (ConcentratIR2; Harrick Scientific Products, New York, USA) comprising a silicon ATR sampling plate optically connected to $\mathrm{ZnSe}$ radiation coupling optics. A liquid nitrogen cooled MCT detector was used for signal recording. Gold nanoparticles were first synthesized and deposited at the ATR crystal surface for $15 \mathrm{~min}$, and the remaining solution containing unreacted tetrachloroaurate acid and AuNPs not sedimented at the ATR surface were removed. A background spectrum of water or deuterium oxide, respectively, on top of the AuNP film present within the evanescent field was acquired. Then, $20 \mu \mathrm{L}$ of BSA solution in water or deuterium oxide were placed into the ATR cell, and measurements were performed by averaging 200 spectra in the spectral window of $4000-800 \mathrm{~cm}^{-1}$ at a spectral resolution of $2 \mathrm{~cm}^{-1}$. Data 
acquisition and processing was performed using the OPUS 6.5 software package (Bruker Optics, Ettlingen, Germany). $10 \mathrm{mmol} \cdot \mathrm{L}^{-1} \mathrm{SDS}$ solution followed by distilled water was used for cleaning the ATR cell and the waveguide surface after each measurement.

For fundamental complementary studies, the synthesis of AuNPs inside the ATR cell was additionally investigated using a BioATR-II system (Bruker Optics, Ettlingen, Germany) also comprising a silicon ATR sampling plate. However, this ATR unit facilitates temperature control and can be sealed in order to avoid evaporation of the tetrachloroauric acid solution during the synthesis.

\section{Analysis of IR spectra}

Data were analyzed using the OPUS 6.5 software package and PeakFit software. Evolution of the synthesis of AuNPs was evaluated by determining the height of the $\delta\left(\mathrm{H}_{2} \mathrm{O}\right)$ bending vibration band at $1644 \mathrm{~cm}^{-1}$. SEIRA spectra were analyzed by analyzing the height of the protein amide I band (1600$1700 \mathrm{~cm}^{-1}$ ).

Fitting of the BSA spectra in the presence and absence of AuNPs was executed as follows, and as described in detail elsewhere [2]. Firstly, water vapor and $\mathrm{CO}_{2}$ interferences were excluded using routines provided by the OPUS software package. Then, spectra were smoothed using a 13-point SavitzkyGolay algorithm. Using PeakFit, the amide I band was deconvoluted via a Gaussian peak-fit model, and second derivatives with $20 \%$ FFT smoothing were calculated for quantitative secondary structure evaluation.

\section{Results and Discussion}

\section{Infrared spectroscopic monitoring of the formation of the SEIRA-active layer}

Gold nanoparticles were directly synthesized inside the ATR liquid cell mediated by the stainless steel comprising the walls of the cell. As previously shown, stainless steel acts as reducing agent spontaneously leading to the formation of AuNPs without any ligand at the surface [8, 9]. While AuNPs are formed, they inherently sediment at the silicon ATR waveguide surface, and their formation can be monitored by the SEIRA effect produced on the water molecules of the surrounding solution in dependence of the number and density of AuNPs present within the evanescent field. As shown in Fig. 1, as AuNPs are increasingly depositing at the waveguide surface an enhancement of the water bending and the $\mathrm{OH}$ stretching bands is observed.

For monitoring the synthesis of the AuNPs, the peak height of the water bending mode was analyzed. Figure 1 shows the peak height of the water bending mode versus time with $t=0$

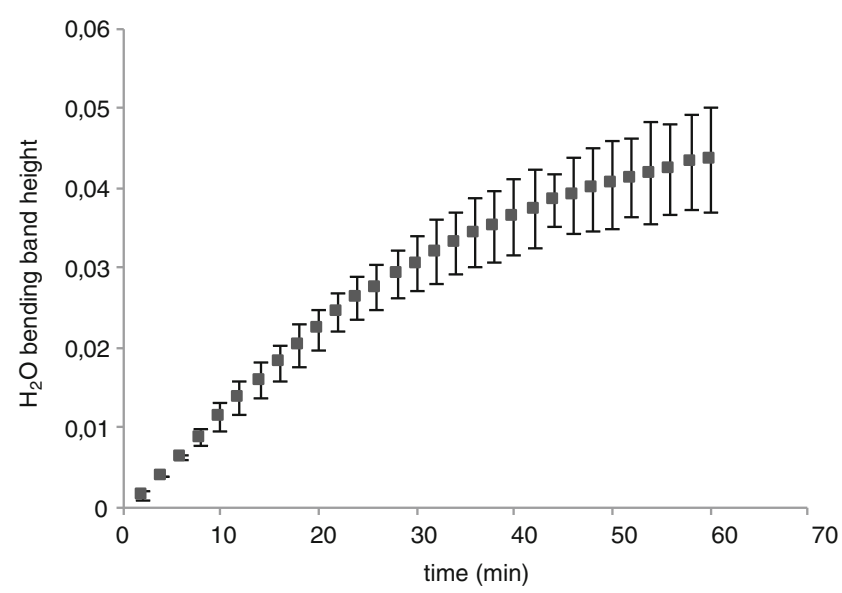

Fig. 1 Evaluation of the SEIRA-ATR spectra obtained during the synthesis of AuNPs inside the ATR unit in aqueous environment. The peak height of the $\delta\left(\mathrm{H}_{2} \mathrm{O}\right)$ bending vibration band at $1644 \mathrm{~cm}^{-1}$ vs. time was evaluated (error bars depict the standard deviation, $n=3$ ). At $t=0$, the $\mathrm{HAuCl}_{4}$ solution was introduced into the ATR liquid cell

indicating the initiation of the AuNP synthesis. The background spectrum was immediately acquired once the tetrachloroauric acid solution was placed into the ATR cell such that the observed signal corresponds to the enhancement produced by the AuNPs as they sediment at the surface vs. the absorption of pure water in the absence of AuNPs.

\section{Characterization of the AuNP SEIRA substrate}

SEIRA substrates formed by the in-situ synthesized AuNP film within the ATR liquid cell were characterized by scanning electron microscopy (SEM). As evident in Fig. 2, AuNPs are of polygonal or spherical shape with an average diameter of $53 \pm 27 \mathrm{~nm}$ calculated by measuring the diameter of a total of 2361 nanoparticles (Fig. S1, see Electronic Supporting Information, ESI). During 15 min of synthesis and sedimentation, the AuNPs are forming a submonolayer coverage at the ATR waveguide with some agglomerates and islands of nanoparticles also present.

\section{Optimization of the $\mathrm{HAuCl}_{4}$ concentration}

The variables that may influence the formation of AuNPs, and consequently, surface enhanced infrared spectroscopy of the protein were analyzed. Firstly, the concentration of the gold precursor, i.e., tetrachloroauric acid solution was investigated in the range between $0.0125-1 \%(w / v)$ (Fig. S2, results shown only until $0.1 \%$ ). All measurements were performed after 15 min of synthesis followed by removal of the supernatant. At low concentrations, a low-density coverage layer of AuNPs is formed at the waveguide surface, which leads to a rather small enhancement of the BSA IR-ATR spectra. At higher concentrations $(>0.5 \%)$, the reaction is apparently too fast, thus leading to the formation of a gold layer at the 
Fig. 2 SEM images of the SEIRA substrate comprising bare (i.e., without ligand) AuNPs synthesized in-situ within the ATR liquid cell taking advantage of stainless steel assisted AuNP generation
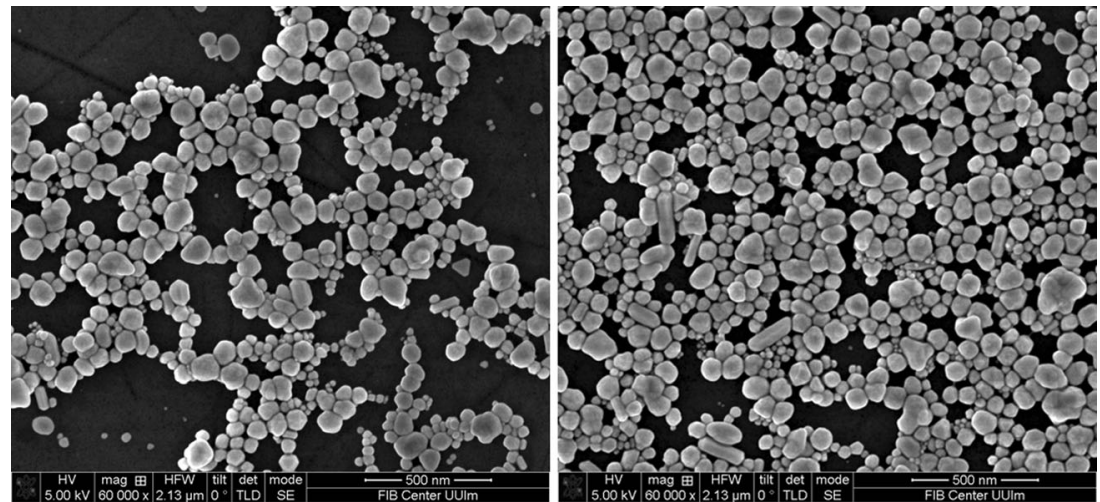

walls of the ATR cell, instead of the homogeneous and reproducible formation of a AuNPs layer at the waveguide surface. Thus, a concentration of $0.1 \%$ of $\mathrm{HAuCl}_{4}$ was selected for further studies on the basis of the enhancement observed, and the reproducibility of the measurements.

\section{Optimization of time of formation for AuNPs}

The time of in-situ synthesis for gold nanoparticles was also evaluated. AuNPs were grown for different periods of time in the ATR liquid cell. As shown in Fig. S3 (ESI), at shorter time periods, only few AuNPs are present within the evanescent field, thus yielding limited signal enhancements, as evidenced by evaluating the height of the BSA amide I band. When increasing the reaction time, more AuNPs are formed, which in turn lead to more pronounced signal enhancements. It has to be noted that these measurements were executed in an ATR unit without top cover, hence, slow evaporation of the aqueous sample may occur. Thus, extended time periods did not lead to further improved signal enhancements, as evident in the inset of Fig. S3, which is potentially attributed to the simultaneous evaporation of the $\mathrm{HAuCl}_{4}$ solution.

If AuNPs are synthesized inside a sealed ATR unit where evaporation does not occur (i.e., the cell is entirely filled with solution without any headspace), the anticipated enhancement of the water absorption bands is observed. First, submonolayer coverage with few agglomerates of AuNPs are observed at the Si wafer surface acting as the ATR waveguide, whereas at longer time periods more pronounced agglomeration of AuNPs and island formation is observed. Evidently, no further increase of the absorption features is occurring, which indicates that the signal enhancement is compensated by the displacement of water molecules from the evanescent field due to AuNPs sedimentation [8] (see Fig. S4, ESI).

AuNPs directly synthesized and deposited on the ATR waveguide are not covalently linked to the surface, thus, before each measurement a new SEIRA substrate is prepared in order to overcome possible irreproducibility due to removal of NPs during the cleaning of the cell. By creating a new substrate, which takes just 15 min and comprises only few microliters of $\mathrm{HAuCl}_{4}$ solution, the ATR liquid cell can be properly cleaned with sodium dodecyl sulfate solution and water in order to eliminate protein before the following measurement.

\section{IR signal enhancement of bare Au nanoparticles}

SEIRA-ATR spectra of BSA at in-situ generated AuNPs and a bare Si ATR waveguide surfaces were compared to evidence signal enhancements resulting from the AuNPs. As illustrated in Fig. 3, two peaks corresponding to the amide I and the amide II band appear at $1600-1700 \mathrm{~cm}^{-1}$ and $1500-1600 \mathrm{~cm}^{-1}$, respectively. The peak at $1652 \mathrm{~cm}^{-1}$ is attributed to the amide I vibration $(\mathrm{C}=\mathrm{O}$ stretching) while the amide II band is associated with the $\mathrm{N}-\mathrm{H}$ stretching vibration along with with $\mathrm{C}-\mathrm{N}$ and $\mathrm{C}=\mathrm{C}$ stretching vibrations [22]. The intensity of the amide I vibration was evaluated in the absence and presence of AuNPs at the ATR waveguide surface revealing an enhancement factor of approx. 4, while - as shown in detail in Fig. 5 - the structure of BSA is maintained.

\section{BSA adsorption at AuNPs}

The adsorption of BSA onto AuNPs was monitored via the evolution of the corresponding SEIRA-ATR spectra. Figure 4 indicates the increase in height of the amide I band of BSA, as it is increasingly adsorbed onto the AuNPs. It should be noted that the background spectrum is BSA protein at AuNPs at $t=0$, thus, only the increase in height of the amide I band by mere accumulation of protein within the evanescent field is observed. As shown in Fig. 4, if a BSA solution is placed in the ATR cell containing AuNPs, an enhancement is immediately observed.

The amide I band entails detailed information on the secondary structure of the protein, as it is related to hydrogen bonds of the peptide chain. Hence, the amide I band is particularly sensitive to structural changes of the 


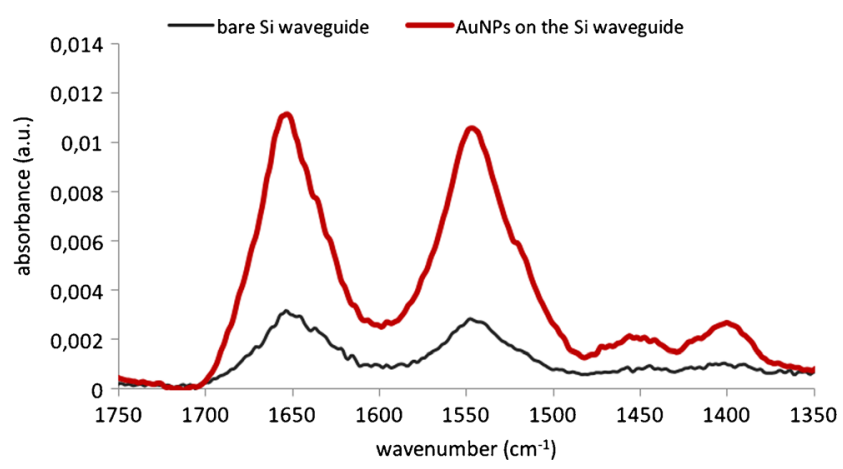

Fig. 3 Normalized IR-ATR spectra of $250 \mathrm{mg} \cdot \mathrm{L}^{-1}$ BSA in water adsorbed onto the AuNPs (red), and protein at the unmodified Si waveguide surface (black)

protein. In order to study if the adsorption of BSA onto AuNPs affects the secondary structure of the protein, a detailed curve fitting of the amide I band of BSA has been performed in the presence and absence of AuNPs (Fig. 5). It is evident that the secondary structure of BSA adsorbed onto AuNPs is readily preserved, thus confirming the potential suitability of the developed IR assay approach for studying the true conformational nature of the protein. The amide I band was evaluated using a Gaussian peak-fit model and second derivatives. In this region, BSA is characterized by peaks at approximately $1655 \mathrm{~cm}^{-1}$, which represent the dominant secondary structure of the $\alpha$-helix [23]. It has to be pointed out that measurements were performed by directly depositing the BSA solution on the ATR liquid cell with the AuNPs on the waveguide surface, without any incubation time. It has been previously reported in literature that adsorption of BSA protein on Au nanoparticles may induce changes in the secondary structure of the protein [20]; however, in most of the studies of adsorption of BSA on AuNPs an incubation time (i.e. 1 h) was used, whereas in our conditions for measurements BSA protein and AuNPs are in contact just few minutes $(\sim 2$ min of the measurement).

\section{SEIRA determination of BSA}

The analytical performance of the method was investigated to evaluate its suitability for quantitative analysis and future label-free protein conformation assays. Enhanced spectra at different concentrations of BSA were recorded at the previously optimized conditions (i.e., $15 \mathrm{~min}$ synthesis of AuNPs inside the ATR liquid cell using a $\mathrm{HAuCl}_{4}$ solution with a concentration of $0.1 \%$ ). Solutions of BSA in water were prepared from a $10 \mathrm{mg} \cdot \mathrm{mL}^{-1}$ stock solution in a range of $25-250 \mathrm{mg} \cdot \mathrm{L}^{-1}$. SEIRA-ATR spectra were acquired upon deposition of BSA protein solution onto the SEIRA substrate. For each measurement, a new AuNPs substrate was in-situ prepared. The analytical signal (i.e., height of the protein amide I band) was plotted against the BSA concentration (Fig. 6). Each point corresponds to the average of three independent measurements, and the error bars illustrate the standard deviation of the corresponding mean value. Evidently, the calibration function can be adjusted to a Langmuir isotherm $\left(\mathrm{R}^{2}=0.99609\right)$, which corresponds to the adsorption process of the protein at the AuNP layer present at the ATR waveguide surface. The corresponding Langmuir equation was $\mathrm{y}=\left(\mathrm{a}^{*} \mathrm{~b} * \mathrm{x}^{\wedge}(1-\mathrm{c})\right) /$ $\left(1+b^{*} x^{\wedge}(1-c)\right)$ fitted by the following parameters: $\mathrm{a}=0.042, \mathrm{~b}=0.0095, \mathrm{c}=0.386$. The limit of detection (LOD), calculated as $\left(3 \mathrm{~S}_{\mathrm{a}}\right) / \mathrm{b}$ for $\mathrm{y}=\mathrm{b} * \log [\mathrm{BSA}]+\mathrm{a}$, after logarithmic transformation of the concentrations according to that described by Massarini et al. [24] was $1.93 \mathrm{mg} \cdot \mathrm{L}^{-1}$. The precision of the measurements was evaluated at a concentration of $250 \mathrm{mg} \cdot \mathrm{L}^{-1}$ yielding a relative standard deviation $(n=5)$ of $11.9 \%$.
Fig. 4 Adsorption isotherm for $125 \mathrm{mg} \cdot \mathrm{L}^{-1}$ BSA solution at AuNPs. AuNPs were synthesized for $15 \mathrm{~min}$, then the BSA solution was placed into the ATR liquid cell, and a background spectrum was recorded. Thus, the peak height illustrates the increase on the amide I signal of BSA, as the protein adsorbs onto the AuNPs

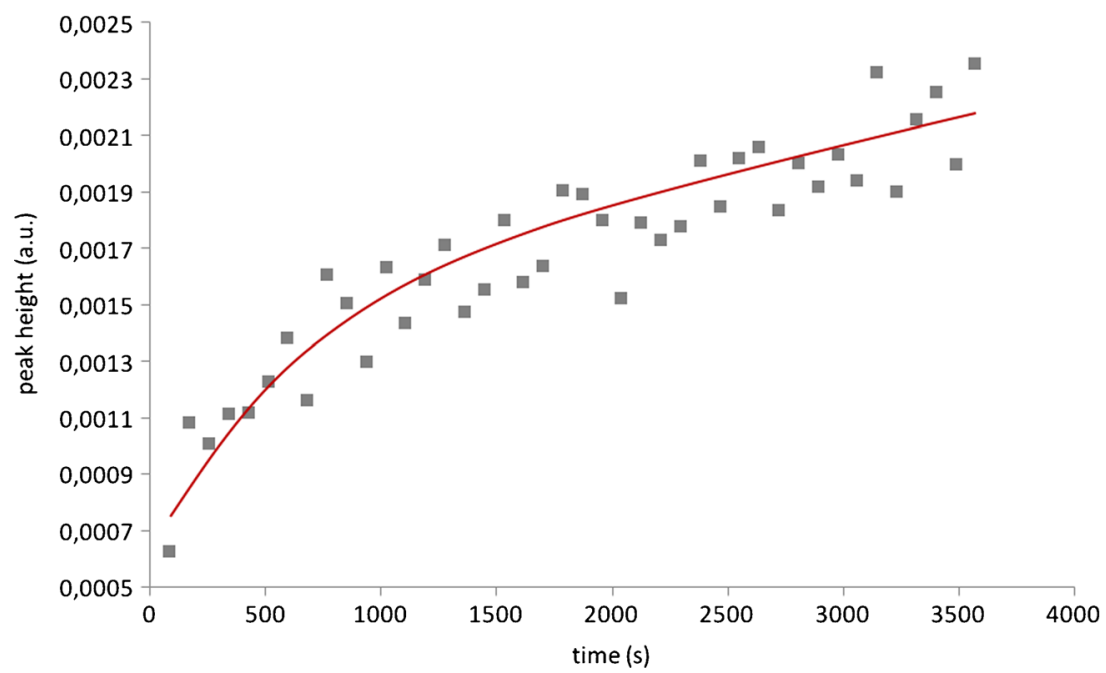



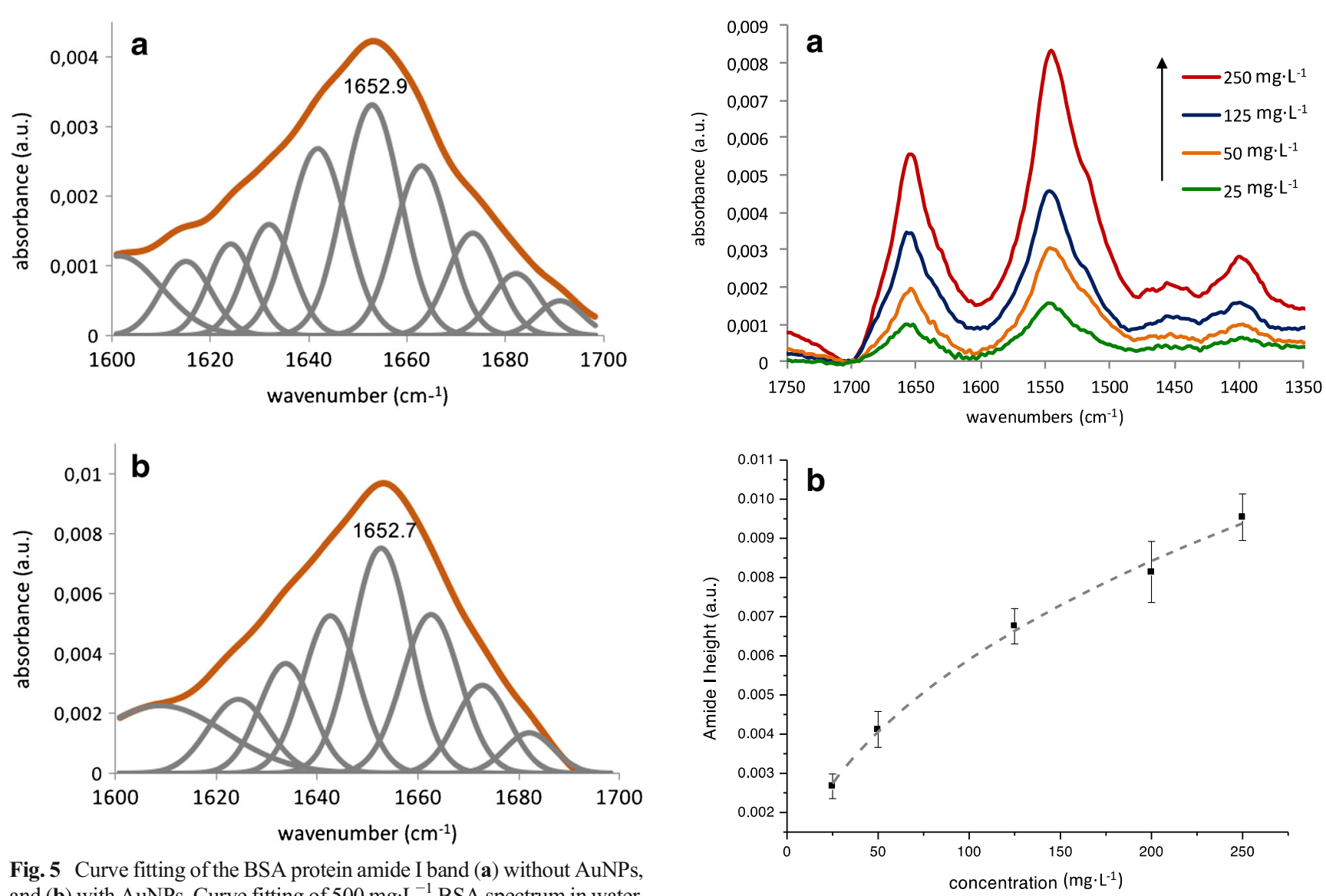

Fig. 5 Curve fitting of the BSA protein amide I band (a) without AuNPs, and (b) with AuNPs. Curve fitting of $500 \mathrm{mg} \cdot \mathrm{L}^{-1} \mathrm{BSA}$ spectrum in water. Spectra were fitted by the Gaussian peak-fitting method

\section{SEIRA studies of BSA in deuterium oxide.}

Additional measurements were performed in deuterium oxide $\left(\mathrm{D}_{2} \mathrm{O}\right)$. Deuterium oxide facilitates the characterization of proteins via IR spectroscopy, as it significantly reduces the strongly absorbing background of water specifically within the spectral regime of interest (i.e., amide I band), while the characteristics of the protein spectra are largely preserved. It should be noted that deuterium substitution causes the amide II band, which usually appears around $1550 \mathrm{~cm}^{-1}$, to shift to a lower wavenumber (approx. $1450 \mathrm{~cm}^{-1}$, amide II') [25]. In addition, an N-H residual amide II peak is present due to the globular nature of the proteins containing labile hydrogens inaccessible to solvent [26].

Spectra were evaluated similar to the studies in aqueous media evaluating the height of the amide I band. Each data point again corresponds to the average of three independent measurements, and the error bars represent the standard deviation of corresponding the mean value. As depicted in Fig. 7, the calibration can again be adjusted to a Langmuir isotherm $\left(\mathrm{R}^{2}=0.99465\right)$, with the fit parameters as: $a=0.401, b=0.001, c=0.433$. The limit of detection ( $L O D)$, calculated as $\left(3 \mathrm{~S}_{\mathrm{a}}\right) / \mathrm{b}$ for $\mathrm{y}=\mathrm{b} * \log [\mathrm{BSA}]+\mathrm{a}$, was $4.15 \mathrm{mg} \cdot \mathrm{L}^{-1}$. The precision of

Fig. 6 a Normalized SEIRA-ATR spectra of BSA at different concentrations ranging from 25 to $250 \mathrm{mg} \cdot \mathrm{L}^{-1}$. b Height of the amide I band from 1600 to $1700 \mathrm{~cm}^{-1}$ in SEIRA-ATR spectra of BSA in aqueous solution vs. concentration. Each point represents the average of 3 independent measurements; error bars relate to the standard deviation of the corresponding mean value. The curve was fitted to a Langmuir isotherm

the measurements was in the range of $3.99-17 \%$ in term of relative standard deviation. Table 1 summarizes the analytical features of the method in both aqueous and deuterium oxide media.

Table 2 shows an overview of other studies reported in literature for investigation of different proteins based on surface enhanced infrared spectroscopy, using both nanostructured surfaces as well as gold surfaces.

\section{Conclusions}

Surface enhanced infrared absorption spectroscopy enabled the determination of BSA protein with enhanced sensitivity. The innovation of the present study is that bare (i.e., without any organic ligand) AuNPs forming the SEIRA substrate were directly and in-situ synthesized within the ATR liquid cell mediated by the stainless steel enclosure, thereby avoiding the use of additional reducing 

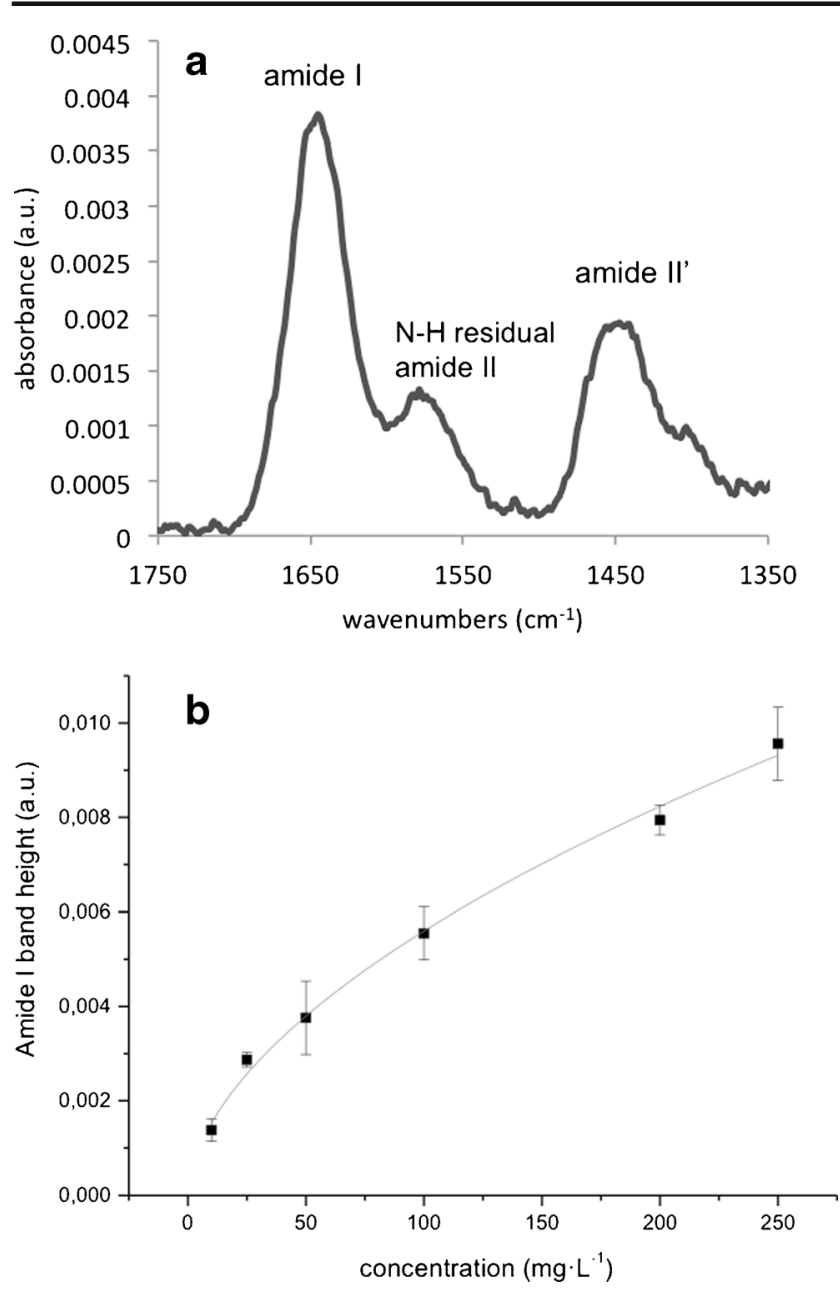

Fig. 7 a IR-ATR spectrum of BSA in $\mathrm{D}_{2} \mathrm{O}$ media at an unmodified $\mathrm{Si}$ ATR waveguide surface ( $500 \mathrm{mg} \cdot \mathrm{L}^{-1}$ of BSA protein). $\mathbf{b}$ Height of the amide I band from 1600 to $1700 \mathrm{~cm}^{-1}$ in SEIRA-ATR spectra in $\mathrm{D}_{2} \mathrm{O}$ vs. concentration of BSA. Each data point represents the average of 3 independent measurements with the error bars illustrating the standard deviation of the corresponding mean value. The curve was fitted to a Langmuir isotherm

reagents that would have to be removed prior to protein analysis. Protein determination is very important for clinical diagnostics purposes, in pathology, immunology, etc.
However, one of the problems of protein determination is the low concentrations of proteins in biological media; thus, methodologies for improving the sensitivity of their determination are demanded, which are usually based on the functionalization of the waveguide surface i.e. with enrichment membranes or recognition elements such as antibodies, aptamers, etc., that allows preconcentration of the protein at the evanescent field. With this approach, in which gold nanoparticles can be in-situ prepared inside the ATR liquid cell within a few minutes, we overcome the functionalization of the internal reflection element. Hence, a strategy for an in-situ renewable SEIRA interface at the ATR waveguide surface is provided that may readily combine with a label-free flow assay arrangement. Nevertheless, in the case of samples such as complex biological fluids (i.e. cerebrospinal fluid) or agrifood samples (e.g. milk, since the content of BSA in milk can be used as a marker of the health of the mammary gland of the cow), further investigations regarding selectivity and the potential use of other recognition elements should be investigated.

BSA proteins are characterized by a pronounced affinity towards AuNPs, which enables the determination of their true secondary structure via enhanced infrared signatures, whereby it was shown that the adsorption of the protein onto the nanoparticles does not modify the secondary structure at the selected measurements conditions. Last but not least, quantitative analysis of BSA protein was demonstrated in both water and deuterium oxide with the latter medium minimizing the background absorption of water within the spectral regime of interest (i.e., the amide I band). Hence, the present study evidences the potential of IR-ATR spectroscopy and sensing schemes as label-free mid-infrared protein conformation assay technology with enhanced sensitivity, which is of particular interest in combination with recently emerging miniaturized mid-infrared sensing schemes based on thin-film waveguide technologies combined with quantum cascades lasers [18, 34-37].
Table 1 Analytical features of the method in water and deuterium oxide media

\begin{tabular}{lll}
\hline & Aqueous medium & Deuterium oxide medium \\
\hline Calibration equation & $\mathrm{y}=\left(\mathrm{a}^{*} \mathrm{~b}^{*} \mathrm{x}^{\wedge}(1-\mathrm{c})\right) /\left(1+\mathrm{b}^{*} \mathrm{x}^{\wedge}(1-\mathrm{c})\right)^{\mathrm{a}}$ & $\mathrm{y}=\left(\mathrm{a}^{*} \mathrm{~b}^{*} \mathrm{x}^{\wedge}(1-\mathrm{c})\right) /\left(1+\mathrm{b}^{*} \mathrm{x}^{\wedge}(1-\mathrm{c})\right)^{\mathrm{b}}$ \\
$\mathrm{R}^{2}$ & 0.99609 & 0.99465 \\
Range studied & $25-250 \mathrm{mg} \cdot \mathrm{L}^{-1}$ & $10-250 \mathrm{mg} \cdot \mathrm{L}^{-1}$ \\
$\mathrm{LOD}^{\mathrm{c}}$ & $1.93 \mathrm{mg} \cdot \mathrm{L}^{-1}$ & $4.15 \mathrm{mg} \cdot \mathrm{L}^{-1}$ \\
$\operatorname{RSD}^{\mathrm{d}}(\%)$ & $11.9 \%$ & $8.1 \%$ \\
\hline
\end{tabular}

a Parameters of the equation: $\mathrm{a}=0.042, \mathrm{~b}=0.0095, \mathrm{c}=0.386$

${ }^{\mathrm{b}}$ Parameters of the equation: $\mathrm{a}=0.401, \mathrm{~b}=0.001, \mathrm{c}=0.433$

${ }^{\mathrm{c}}$ Limit of detection, determined as $\left(3 \mathrm{~S}_{\mathrm{a}}\right) / \mathrm{b}$, for $\mathrm{y}=\mathrm{b} * \log [\mathrm{BSA}]+\mathrm{a}$

${ }^{\mathrm{d}}$ Relative standard deviation, determined from the average value of $n=5$ at a concentration of $250 \mathrm{mg} \cdot \mathrm{L}^{-1}$ of BSA 


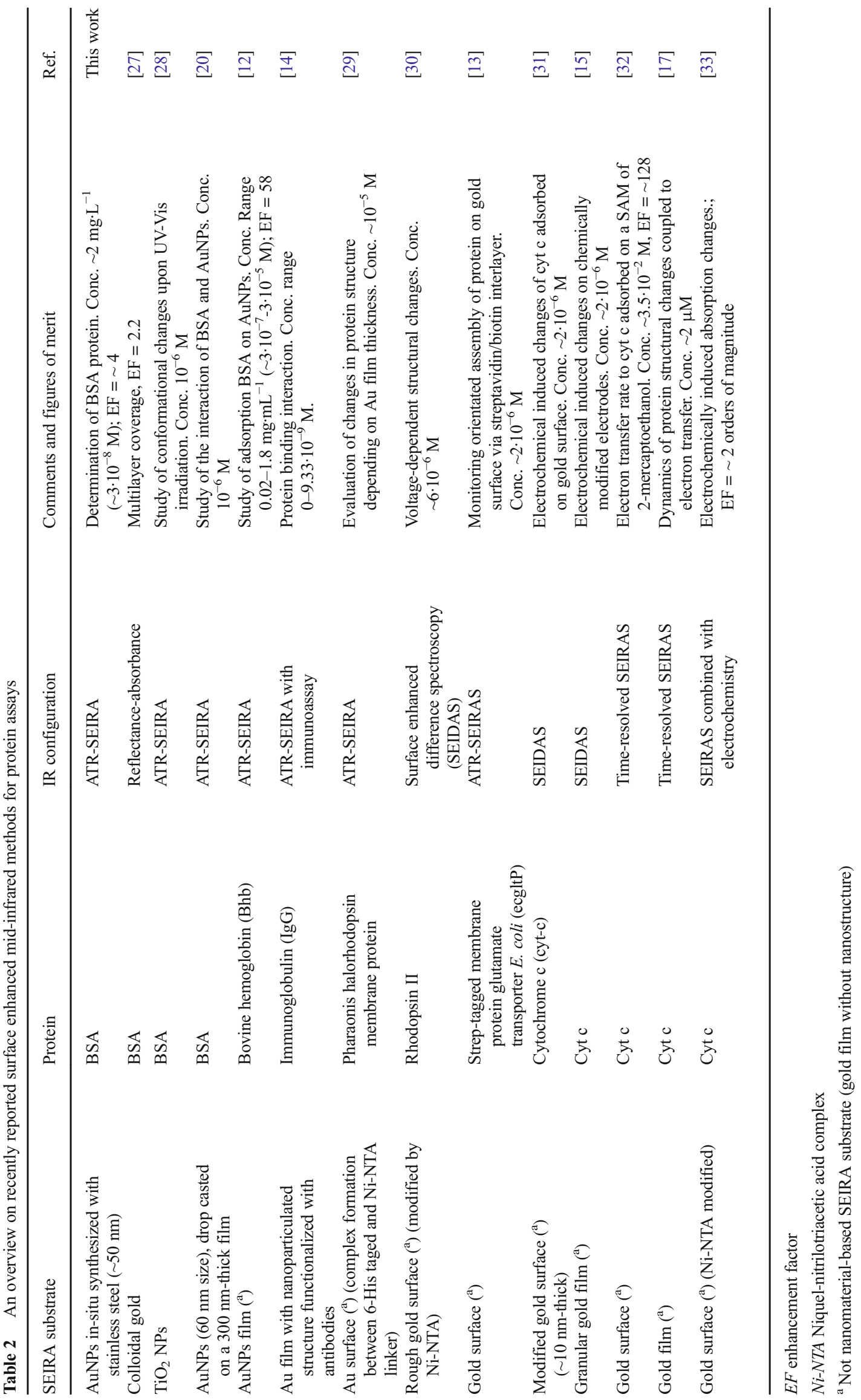


Acknowledgements A.I. López-Lorente wishes to thank the Alexander von Humboldt Foundation for the award of a Postdoctoral Fellowship at the Institute of Analytical and Bioanalytical Chemistry (University of Ulm, Germany). This work was in part supported by the Boehringer Ingelheim Ulm University BioCenter (BIU), and by the International Graduate School in Molecular Medicine at the University of Ulm, which provided a graduate student fellowship. Funding by the Horizon 2020 Framework Program of the European Union within the MSCA RISE Project TROPSENSE is also acknowledged.

Compliance with ethical standards The author(s) declare that they have no competing interests.

\section{References}

1. López-Lorente AI, Mizaikoff B (2016) Mid-infrared spectroscopy for protein analysis: potential and challenges. Anal Bioanal Chem 408:2875-2889

2. Wang P, Bohr W, Otto M, Danzer KM, Mizaikoff B (2015) Quantifying amyloid fibrils in protein mixtures via infrared attenuated-total-reflection spectroscopy. Anal Bioanal Chem 407: 4015-4021

3. Harstein A, Kirtley JR, Tsang JC (1980) Enhancement of the infrared absorption from molecular monolayers with thin metal Overlayers. Phys Rev Lett 45:201-204

4. Osawa M (2001) Surface-enhanced infrared absorption. Top Appl Phys 81:163-187

5. Ataka K, Heberle J (2007) Biochemical applications of surfaceenhanced infrared absorption spectroscopy. Anal Bioanal Chem $388: 47-54$

6. Le F, Brandl DW, Urzhumov YA, Wang H, Kundu J, Halas NJ, Aizpurua J, Nordlander P (2008) Metallic nanoparticle arrays: a common substrate for both surface-enhanced Raman scattering and surface-enhanced infrared absorption. ACS Nano 2:707-718

7. Enders D, Nagao T, Pucci A, Nakayama T, Aono M (2011) Surfaceenhanced ATR-IR spectroscopy with interface-grown plasmonic gold-island films near the percolation threshold. Phys Chem Chem Phys 13:4935-4941

8. López-Lorente AI, Sieger M, Valcárcel M, Mizaikoff B (2014) Infrared attenuated Total reflection spectroscopy for the characterization of gold nanoparticles in solution. Anal Chem 86:783-789

9. López-Lorente AI, Simonet BM, Valcárcel M, Eppler S, Schindl R, Kranz C, Mizaikoff B (2014) Characterization of stainless steel assisted bare gold nanoparticles and their analytical potential. Talanta 118:321-327

10. López-Lorente AI, Valcárcel M, Mizaikoff B (2014) Continuous flow synthesis and characterization of tailor-made bare gold nanoparticles for use in SERS. Microchim Acta 181:1101-1108

11. López-Lorente AI, Simonet BM, Valcárcel M, Mizaikoff B (2013) Bare gold nanoparticles mediated surface-enhanced Raman spectroscopic determination and quantification of carboxylated singlewalled carbon nanotubes. Anal Chim Acta 788:122-128

12. Jin B, Bao WJ, Wu ZQ, Xia XH (2012) In situ monitoring of protein adsorption on a nanoparticulated gold film by attenuated Total reflection surface-enhanced infrared absorption spectroscopy. Langmuir 28:9460-9465

13. Jiang X, Zuber A, Heberle J, Ataka K (2008) In situ monitoring of the orientated assembly of strep-tagged membrane proteins on the gold surface by surface enhanced infrared absorption spectroscopy. Phys Chem Chem Phys 10:6381-6387

14. Xu JY, Chen TW, Bao WJ, Wang K, Xia XH (2012) Label-free strategy for In-situ analysis of protein binding interaction based on attenuated Total reflection surface enhanced infrared absorption spectroscopy (ATR-SEIRAS). Langmuir 28:17564-17570

15. Ataka K, Heberle J (2004) Functional vibrational spectroscopy of a cytochrome $\mathrm{c}$ monolayer: SEIDAS probes the interaction with different surface-modified electrodes. J Am Chem Soc 126:9445-9457

16. Ataka K, Giess F, Knoll W, Naumann R, Haber-Pohlmeier S, Richter B, Heberle J (2004) Oriented attachment and membrane reconstitution of his-tagged cytochrome c oxidase to a gold electrode: in situ monitoring by surface-enhanced infrared absorption spectroscopy. J Am Chem Soc 126:16199-16206

17. Wisitruangsakul N, Zebger I, Ly KH, Murgida DH, Ekgasit S, Hildebrandt P (2008) Redox-linked protein dynamics of cytochrome c probed by time-resolved surface enhanced infrared absorption spectroscopy. Phys Chem Chem Phys 10:5276-5286

18. Lu R, Li WW, Katzir A, Raichlin Y, Yu HQ, Mizaikoff B (2015) Probing the secondary structure of bovine serum albumin during heat-induced denaturation using mid-infrared fiberoptic sensors. Analyst 140:765-770

19. Tsai DH, Davila-Morris M, DelRio FW, Guha S, Zachariah MR, Hackley VA (2011) Quantitative determination of competitive molecular adsorption on gold nanoparticles using attenuated Total reflectance!Fourier transform infrared spectroscopy. Langmuir 27: 9302-9313

20. Tsai DH, DelRio FW, Keene AM, Tyner KM, MacCuspie RI, Cho TJ, Zachariah MR, Hackley VA (2011) Adsorption and conformation of serum albumin protein on gold nanoparticles investigated using dimensional measurements and in situ spectroscopic methods. Langmuir 27:2464-2477

21. Brewer SH, Glomm WR, Johnson MC, Knag MK, Franzen S (2005) Probing BSA binding to citrate-coated gold nanoparticles and surfaces. Langmuir 21:9303-9307

22. Moulton SE, Barisci JN, McQuillan AJ, Wallace GG (2003) ATRIR spectroscopic studies of the influence of phosphate buffer on adsorption of immunoglobulin $\mathrm{G}$ to $\mathrm{TiO}_{2}$. Colloids Surf A Physicochem Eng Asp 220:159-167

23. Jackson M, Mantsch HH (1995) The use and misuse of FTIR spectroscopy in the determination of protein structure. Crit Rev Biochem Mol Biol 30:95-120

24. Massarini E, Wästerby $\mathrm{P}$, Landström L, Lejon C, Beck O, Andersson PO (2015) Methodologies for assessment of limit of detection and limit of identification using surface-enhanced Raman spectroscopy. Sensors Actuators B 207:437-446

25. Militello V, Casarino C, Emanuele A, Giostra A, Pullara F, Leone M (2004) Aggregation kinetics of bovine serum albumin studied by FTIR spectroscopy and light scattering. Biophys Chem 107:175187

26. Lad MD, Birembaut F, Matthew JM, Frazier RA, Green RJ (2006) The adsorbed conformation of globular proteins at the air/water interface. Phys Chem Chem Phys 8:2179-2186

27. Dovbeshko G, Fesenko O, Nazariva A (2006) Effect of nanostructured metal surface on SEIRA spectra of albumin and nucleic acids. J Phys Stud 10:127-134

28. Bouhekka A, Bürgi T (2012) In situ ATR-IR spectroscopy study of adsorben protein: visible light denaturalization of bovine serum albumin on TiO2. Appl Surf Sci 261:369-374

29. Guo H, Kimura T, Furutani Y (2013) Distortion of the amide-I and II bands of an $\alpha$-helical membrane protein, pharaonis halorhodopsin, depends on thickness of gold films utilized for surface-enhanced infrared absorption spectroscopy. Chem Phys 419:8-16

30. Jiang X, Zaitseva E, Schmidt M, Siebert F, Engelhard M, Schlesinger R, Ataka K, Vogel R, Heberle J (2008) Resolving voltage-dependent structural changes of a membrane photoreceptor by surface-enhanced IR difference spectroscopy. Proc Natl Acad Sci 105:12113-12117 
31. Ataka K, Heberle J (2003) Electrochemically induced surfaceenhanced infrared difference absorption (SEIDA) spectroscopy of a protein monolayer. J Am Chem Soc 125:4986-4987

32. Nowak C, Luening C, Schach D, Baurecht D, Knoll W, Naumann RLC (2009) Electron transfer kinetics of cytochrome $\mathrm{C}$ in the Submillisecond time regime using timeresolved surface-enhanced infrared absorption spectroscopy. J Phys Chem C 113:2256-2262

33. Ataka K, Heberle J (2006) Use of surface enhanced infrared absorption spectroscopy (SEIRA) to probe the functionality of a protein monolayer. Biopolymers 82:415-419

34. Sieger M, Haas J, Jetter M, Michler M, Godejohann M, Mizaikoff B (2016) Mid-infrared spectroscopy platform based on GaAs/
AlGaAs thin-film waveguides and quantum Cascade lasers. Anal Chem 88:2558-2562

35. Wang X, Karlsson M, Sieger M, Österlund L, Nikolajeff F, Mizaikoff B (2014) Diamonds are a Spectroscopist's best friend: thin-film diamond mid-infrared waveguides for advanced chemical sensors/biosensors. Anal Chem 86:8136-8141

36. López-Lorente AI, Wang P, Sieger M, Vargas Catalan E, Karlsson M, Nikolajeff F, Österlund L, Mizaikoff B (2016) Mid-infrared thin-film diamond waveguides combined with tunable quantum cascade lasers for analyzing the secondary structure of proteins. Phys Status Solidi A. doi:10.1002/pssa.201600134

37. Mizaikoff B (2013) Waveguide-enhanced mid-infrared chem/bio sensors. Chem Soc Rev 42:8683-8699 